\title{
The Mediating Effects of Self-Esteem and Optimism on the Relationship between Quality of Life and Depressive Symptoms of Breast Cancer Patients
}

\author{
Eun Hye $\mathrm{Ha}^{\bowtie}$ and Young Kyung Cho \\ Department of Child Welfare and Child Studies, Sookmyung Women's University, Seoul, Republic of Korea
}

Objective This study investigated the mediating effects of the internal psychological factors of self-esteem and optimism on the relationship between breast cancer patients' quality of life in terms of symptoms and functioning and depressive symptoms.

Methods The study centered on 384 breast cancer patients who had within a 24-month period received diagnosis of 0-4 stage cancer and had medical treatment. To achieve the study's purpose, the study made use of EORTC QLQ BR23, CES-D, and the Self-Esteem and Optimism Scales.

Results Findings revealed that breast cancer patients' quality of life was negatively impacted by self-esteem and optimism, and that self-esteem and optimism impacted negatively on depressive symptoms. Analyses showed that when breast cancer patients' quality of life affects depressive symptoms, the full mediation effect of self-esteem was statistically significant. Also, findings revealed there to be a significant partial mediation effect due to optimism.

Conclusion Study findings demonstrated that enhancing self-esteem is crucial in the psychological intervention of depressive symptoms because self-esteem functioned as the main causal factor accounting for all variation when breast cancer patients' quality of life affected depressive symptoms. In addition, results suggested that optimism is also vital to psychological intervention because it functioned as partial cause of heightened depressive symptoms when breast cancer patients' quality of life affected depressive symptoms.

Psychiatry Investig 2014;11(4):437-445

Key Words Breast neoplasms, Quality of life, Depression, Self-Esteem, Optimism.

\section{INTRODUCTION}

Depression is the most commonly occurring psychological disorder among cancer patients. Comorbidity of depression with breast cancer reaches as high as $46 \%,{ }^{1}$ and in the year when breast cancer is diagnosed, patients are most vulnerable to the illness. In fact, even 5 years after a diagnosis, about 15\% of patients experience depressive disorders. ${ }^{2}$ Because depressive symptoms of breast cancer patients are associated with various psychological problems, in addition to negatively af-

Received: August 7, 2013 Revised: November 26, 2013

Accepted: February 4, 2014 Available online: October 20, 2014

$\triangle$ Correspondence: Eun Hye $\mathrm{Ha}, \mathrm{PhD}$

Department of Child Welfare and Child Studies, Sookmyung Women's University, 100 Cheongpa-ro 47-gil, Yongsan-gu, Seoul 140-742, Republic of Korea

Tel: +82-2-2077-7176, Fax: +82-2-710-9209

E-mail: graceha@sookmyung.ac.kr

(a) This is an Open Access article distributed under the terms of the Creative Commons Attribution Non-Commercial License (http://creativecommons.org/licenses/by$\mathrm{nc} / 3.0$ ) which permits unrestricted non-commercial use, distribution, and reproduction in any medium, provided the original work is properly cited. fecting psychosocial adjustment, they negatively affect overall quality of life, which reduces survival rates due to a decrease in therapeutic compliance. ${ }^{3}$ Moreover, because depression among breast cancer patients worsens prognosis by weakening the will to fight and decreasing immunity, early screening of breast cancer patients for depression and psychosocial intervention is essential.

Physical symptoms and functionings that lower breast cancer patients' quality of life have also been shown to seriously influence on depressive symptoms. ${ }^{4}$ In a study on the effect of disease-related factors and health-related quality of life on depressive symptoms, depressive symptoms were found to affect factors such as physical well-being, social roles, affective functioning, pain, sleep disorders, and vomiting. ${ }^{5}$ In a related study on the effect of two sub-factors of health-related quality of life: body image and sexual functioning, 155 breast cancer patients identified body image as the variable influencing their depressive symptoms. ${ }^{6}$ Another study on 49 patients within 4 weeks of surgery ${ }^{7}$ found that depressive symptoms were more 
severe among subjects with poorer body images, more severe breast symptoms, and more severe side effects from full body treatment. In a recent study of breast cancer patients' healthrelated quality of life in terms of physical symptoms and functioning on depressive symptoms, results concluded that the effect of four functioning levels-body image, sexual functioning, sexual interest, and future prospect-along with the effect of four symptom levels-arm symptoms, chest symptoms, side effects, and hair symptoms-accounted for $57 \%$ of all depressive symptoms. In addition, depressive symptoms were found to be more prominent among patients 1) with poorer body images and more pessimistic future prospects, 2) showing the aforesaid 4 functioning levels, and 3) with more various and severe breast symptoms and treatment side effects. ${ }^{8}$

Even though many researches clearly illustrate how breast cancer patients' quality of life directly influences depressive symptoms, not all breast cancer patients who experienced physical symptoms and deterioration of functioning undergo depressive symptoms. Thus, it is important to identify variables that intervene in breast cancer patients' depressive symptoms and internal psychological variables that influence both the quality of life and depressive symptoms such as self-esteem, optimism, and spousal support, which are widely recognized variables.

Self-esteem is a personal evaluation of self-worth and has great way on one's quality of life. ${ }^{9}$ Also, for breast cancer patients, physical change due to a mastectomy has been shown to damage self-esteem and negatively influences body image, but if body image is positive, self-esteem could increase. ${ }^{10,11}$ Berterö ${ }^{9}$ argues that breast cancer patients' maintenance of self-esteem should be supported to enhance their quality of life, based on the notion that self-esteem plays a significant role in the quality of life.

Optimism connotes the attitude of generalizing positive expectations of the future and the ability to mediate stress through perceived positive or negative results. ${ }^{12}$ Long-term study tracking of patients diagnosed with cancer showed pessimistic patients to have higher recurrence and mortality rates. In addition, a study that compared optimism and pessimism in women who had received early diagnosis of breast cancer concluded that breast cancer patients possessing higher optimism had higher life satisfaction and better prognosis. ${ }^{13}$ Moreover, it has been shown that breast cancer patients' level of optimism and psychosocial adjustment are predictors of psychosocial adjustment. ${ }^{14}$ In other words, the higher the breast cancer patient's optimism, the more task-oriented coping they used..$^{15}$ Optimism helps breast cancer patients use effective coping strategies in various problematic situations that reduce their quality of life.

Self-esteem and optimism have positive correlations with breast cancer patients' quality of life, but these two personality qualities are low among people with depressive disorders than among the general population. ${ }^{16,17}$ The possibility of a depressive disorder was found to be particularly high among patients that lack optimism.

Based on the results of earlier studies that self-esteem and optimism are internal psychological variables that influence breast cancer patients' quality of life and depressive symptoms, this study investigated the mediating effects of two internal psychological variables-self-esteem and optimism-when breast cancer patients' quality of life due to physical symptoms and functioning influences depressive symptoms.

\section{METHODS}

\section{Patient selection}

Subjects were outpatients who received diagnosis of breast cancer and had had surgery at one of these three cancer centres: the Breast Cancer Clinic in Y University Hospital, the Breast Cancer Center in S University Hospital, or the National Cancer Center. Subject selection criteria were as follows: 1) breast cancer patients with 0-4 stage cancer, 2) patients within 24 months of their first diagnosis of breast cancer and postsurgery treatment, 3) patients with no recurrence or metastasis to other parts, 4) patients not suffering other chronic diseases, and 5) patients who agreed to participate in the study. This study received approval from the Institutional Review Board (IRB) in the Korean National Cancer Center (No. NCCNCS-09-259). Using the above selection criteria, $384 \mathrm{pa}-$ tients who had given written informed consent were asked to complete a questionnaire, which was distributed to each subject at an outpatient visit. The purpose and procedure of the study were explained before onset of the study. Demographic and medical characteristics of subjects are detailed in Table 1.

\section{Instrument measure}

\section{Subject demographic and clinical characteristics}

Subjects were asked about age, marital status, education lev$\mathrm{el}$, and socioeconomic level. They were also questioned about the type of medical treatment they had received (chemotherapy, radiation therapy, hormone therapy), the completion of treatments, the type of surgery (complete resection or partial resection), the elapsed postoperative period, family history of breast cancer, and their primary caregiver.

\section{The European Organization for Research and Treatment of Cancer Quality of Life Questionnaire - Breast Cancer Module}

In order to measure breast cancer patients' quality of life at 
Table 1. Demographic and clinical characteristics of study subjects $(N=384)$

\begin{tabular}{lccc}
\hline \multicolumn{1}{c}{ Characteristic } & $\mathrm{N}(\%)$ & Characteristic & $\mathrm{N}(\%)$ \\
\hline Age & $5(1.3)$ & Disease stage & $22(5.9)$ \\
$20-29$ & $62(16.1)$ & 0 & $155(41.9)$ \\
$30-39$ & $185(48.2)$ & $\mathrm{II}$ & $143(38.7)$ \\
$40-49$ & $103(26.8)$ & $\mathrm{III}$ & $48(13.0)$ \\
$50-59$ & $29(7.6)$ & IV & $2(0.5)$ \\
$60-69$ & & Monthly Income (in millions of Korean Won $)$ & \\
Education level & $59(15.5)$ & $<2$ & $136(23.9)$ \\
Middle school or lower & $174(45.6)$ & $2-3.99$ & $88(23.5)$ \\
High school & $148(38.9)$ & $4-5.99$ & $60(16.1)$ \\
College or higher & & $\geq 6$ & $28(7.3)$ \\
& & Marital status & $310(80.9)$ \\
Family history & $43(11.3)$ & Single & $12(3.1)$ \\
Yes & $339(88.7)$ & Married & $25(6.5)$ \\
No & & Divorced & $6(1.7)$ \\
Type of surgery & $265(69.2)$ & Widowed & $2(0.5)$ \\
Partial mastectomy & $96(25.1)$ & Separated & De Facto Marriage \\
Total mastectomy & &
\end{tabular}

the functioning and symptom levels, the study utilized Yun et al.s ${ }^{18}$ validated The European Organization for Research and Treatment of Cancer Quality of Life Questionnaire - Breast Cancer Module (EORTC QLQ-BR23) on quality of life. ${ }^{19}$ The EORTC scale consists of 23 items. Eight items measure functioning levels pertaining to four areas: body image, sexual functioning, sexual enjoyment, and future prospect while the other fifteen items evaluate symptoms such as the side effects of full body treatment, breast symptoms, arm symptoms, and anxiety due to hair loss. The Cronbach a for this study ranges from $0.72-0.91$.

After questionnaire item completion, patient scores are converted to the 100-point scale specified by the EORTC QLQC30 Scoring Manual. ${ }^{19}$ A high score on the functioning scale and a low score on the symptom scale imply high quality of life. In order to obtain a total score for quality of life for the two opposing scales, scores from symptom scale items were converted to positive scores following the EORTC QLQ-C30 Scoring Manual.

\section{Center for Epidemiological Studies-Depressions}

This research employed Cho and Kim's ${ }^{20}$ adapted questionnaire from the instrument developed by the National Institute of Mental Health (NIMH) for the epidemiological study of depressive syndromes for the general population. It is a self-reporting questionnaire with a total of 20 items. Each item is rated on a scale from 0 (never) to 3 (everyday) depending on frequency throughout the previous week. The possible range for an individual is 0 to 60 points. Chon and Rhee, ${ }^{21}$ from factor analysis, determined 4 final sub-factors: depressive affect, positive affect, interpersonal relationships, and physical deterioration. The Cronbach a for this study is 0.92 .

\section{Self-esteem scale}

The self-esteem scale developed by Rosenberg ${ }^{22}$ and adapted by Jeon ${ }^{23}$ consists of 10 items: 5 items on positive self-esteem and 5 items on negative self-esteem. It is a 4-point Likert scale in which the negative items are scored reversely, with higher scores denoting higher self-esteem. For this study, the Cronbach a internal consistency is $0.77-0.88$ and test-retest reliability is $\mathrm{r}=0.82-0.85$.

\section{Optimism Scale (Life Orientation Test: LOT-R)}

The Life Orientation Test created by Scheider and Carver ${ }^{12}$ and adapted by Park and Son ${ }^{24}$ is used in this study. The total number of test items is 12: 4 positive, 4 negative, and 4 neutral items. It follows a 5-point Likert scale from 1 (Not at all) to 5 (Strongly agree) and has an internal consistency of Cronbach $\alpha=0.83$.

\section{Statistical analysis}

Data were analyzed using SPSS 17.0 and AMOS 18.0. Frequency analysis was also conducted to examine the demographic and disease-related characteristics of the subjects. Moreover, in order to wholly evaluate the mediating effects of selfesteem and optimism when breast cancer patients' quality of life, with regard to physical symptoms and functioning, influences depressive symptoms, structural equation modeling was 


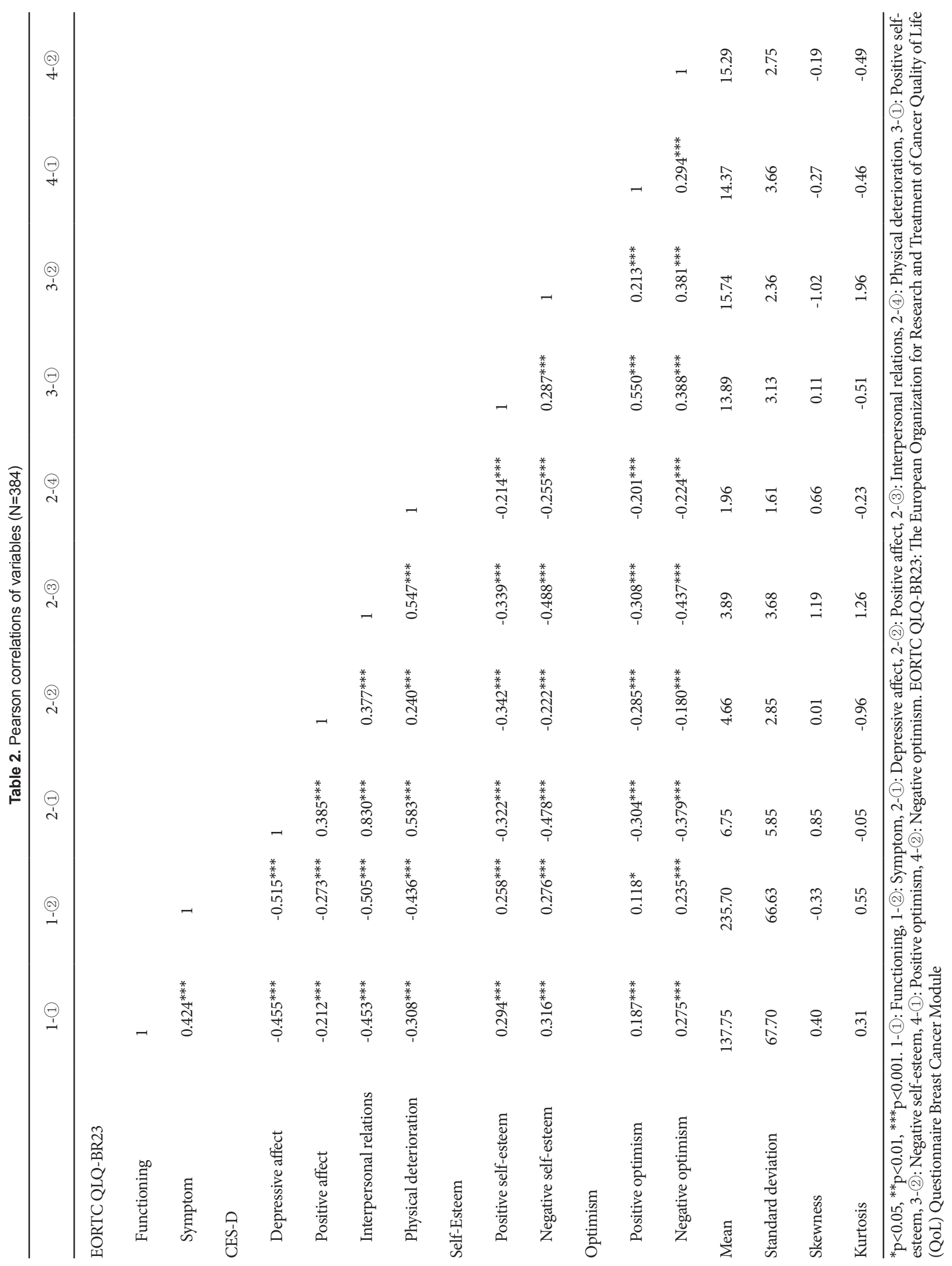


conducted. Structural equation modeling validated the observed model and its corresponding theoretical structural model. It was also tested the relationship between latent variables while controlling measurement errors, which advanced understanding of the mediating effect. Last, the research proposed model was validated for structural model fit using $\chi^{2}$, the Tucker Lewis Index (TLI), the Comparative Fit Index (CFI), and the Root mean square error of approximation (RMSEA).

\section{RESULTS}

\section{Correlations among observed variables}

Correlations among observed variables in terms of mean, standard deviation, and the results of skewness and kurtosis analyses are shown in Table 2. The test of multivariate normal distribution for the 10 observed variables revealed absolute values for skewness to be no more than 2 and absolute values for kurtosis to be no greater than 7 thereby satisfying the assumption for multivariate normal distribution. ${ }^{25}$

\section{Measurement model fit}

In order to determine if observed variables adequately measured respective latent variables, the measurement model was tested. A theoretical variable (latent variable) for quality of life consists of two observed variables: one for quality of life in terms of functioning and symptom and another for 4 observed depressive symptoms, which are depressive affect, positive affect, interpersonal relations, and physical deterioration. The mediating variables of self-esteem and optimism are comprised of two observable aspects, which are the positive and negative factors of each variable. Fit validation test findings for the observed model are reliable; that is, analysis found the observed model to be statistically reliable at $\chi^{2}=108.189$, with $\mathrm{TLI}=0.890, \mathrm{CFI}=0.942$, and $\mathrm{RMSEA}=0.08$.

\section{Structural model fit test for the test of mediating effect and parameter estimation}

A structural model was created to analyze the effect of quality of life on depressive symptoms when mediated by self-esteem and optimism. Analysis of the effect of quality of life on depressive symptoms mediated by self-esteem yielded partial mediated fit $\left(\chi^{2}=40.619\right.$, with TLI $=0.955$, CFI $=0.979$, and RMSEA $=0.06$ ) indicating the study model was reliable (Table 3 ).

The model was then evaluated as a fully mediated model by removing the direct path from predictor of quality of life and adding a direct path to the criterion of depression. The fit of this fully mediated model was good $\left(\chi^{2}=42.563\right.$, with a TLI of 0.956, a CFI of 0.978, and a RMSEA of 0.06) (Table 3). A $\chi^{2}$ difference test was used to examine difference in fit indices between the partial and full mediation models. The test found $\chi^{2}(1)=1.944(p>0.05)$ implying that differences in fit indices between the two models were not statistically significant. The path coefficient for the full mediating model of self-esteem can be found in Table 4 . The path coefficient from quality of life to self-esteem is 0.89 and from self-esteem to depressive symptom is -0.92 . With these statistically significant results, a simplified model of the full model was adopted by researchers (Figure 1).

Analysis of the effect of quality of life on depressive symptoms mediated by optimism yielded a partially mediated model fit of $\chi^{2}=35.127$, with a TLI of 0.964 , a CFI of 0.983 , and a RMSEA of 0.053 , indicating good model fit. When the mod-

Table 3. Fit indices for competing models $(\mathrm{N}=384)$

\begin{tabular}{llllllc}
\hline & \multicolumn{1}{c}{ Model } & $\chi^{2}$ & df & TLI & CFI & RMSEA \\
\hline Self-esteem & Partial mediation model & 40.619 & 17 & 0.955 & 0.979 & 0.060 \\
& Full mediation model & 42.563 & 18 & 0.956 & 0.978 & 0.060 \\
\multirow{3}{*}{ Optimism } & Partial mediation model & 35.127 & 17 & 0.964 & 0.983 & 0.053 \\
& Full mediation model & 45.463 & 18 & 0.948 & 0.974 & 0.063 \\
\hline
\end{tabular}

$\chi^{2}$ : chi-squared, df: degrees of freedom, TLI: Tucker-Lewis Index, CFI: Comparative Fit Index, RMSEA: Root Mean Square Error of Approximation

Table 4. Path Coefficient of the final model $(\mathrm{N}=384)$

\begin{tabular}{llcccc}
\hline & \multicolumn{1}{c}{ Path } & Unstandardized & S.E & C.R & Standardized \\
\hline Full of self-esteem & QoL $\rightarrow$ Self-esteem & 0.03 & 0.01 & $6.03^{* * *}$ & 0.89 \\
& Self-esteem $\rightarrow$ Depressive symptom & -3.71 & 0.51 & $-7.29^{* * *}$ & -0.92 \\
& QoL $\rightarrow$ Depressive symptom & - & - & - & - \\
Partial of optimism & QoL $\rightarrow$ Optimism & 0.02 & 0.01 & $4.64^{* * *}$ & 0.57 \\
& Optimism $\rightarrow$ Depressive symptom & -1.14 & 0.35 & $-3.29^{* *}$ & -0.37 \\
& QoL $\rightarrow$ Depressive symptom & -0.07 & 0.01 & $-5.67^{* * *}$ & -0.61 \\
\hline
\end{tabular}

${ }^{* *} \mathrm{p}<0.01,{ }^{* * *} \mathrm{p}<0.001$. QoL: Quality of life 
el was tested as a fully mediated model by removing the direct path from predictor of quality of life and adding a direct path to the criterion of depression, fit indices of the fully mediated model were $\chi^{2}=45.463$, TLI $=0.948, \mathrm{CFI}=0.974$, and RM$\mathrm{SEA}=0.063$. These results revealed that this model, too, had good fit (Table 3 ). However, the $\chi^{2}$ difference test between the partial and full mediation models resulted in $\chi^{2}(1)=10.336$ $(p<0.05)$ implying that difference in fit indices between the two models was statistically significant. The path coefficient for the partial mediating model of optimism is shown in Table 4. A path coefficient from quality of life to optimism is 0.57 , from optimism to depressive symptom is -0.37 , and from quality of life to depressive symptom is -0.61 . These statistically significant findings led researchers to adopt the partially mediated model with a direct path from predictor of quality of life (Figure 2).

\section{DISCUSSION}

The present study analyzed the mediating effects of self-esteem and optimism on breast cancer patients' quality of life as it affects depressive symptoms using structural equation modeling. Implications and discussion of the effects to breast cancer patients' quality of life based on physical symptoms and functioning in terms of depressive symptoms mediated by self-esteem and optimism will be detailed next. Discussion will also include analysis results attained from the quality of life scale, EORTC QLQ-BR23.

First, correlation analysis of breast cancer patients' quality of life and depressive symptoms indicated that deterioration of
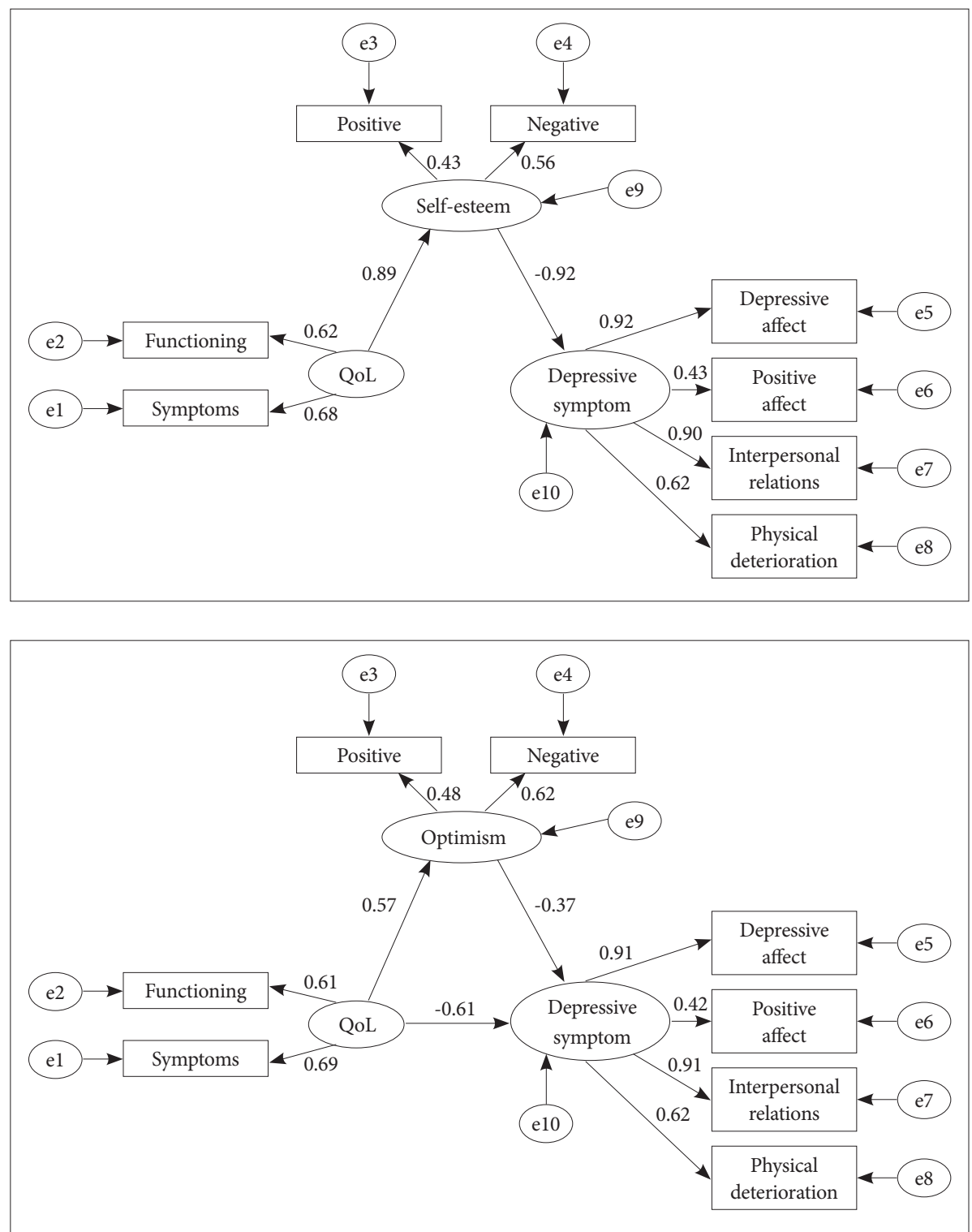

Figure 1. Full mediation model (standardized coefficients) standardized regression coefficients are shown next to each path. QoL: quality of life (EORTC QLQ-BR23).
Figure 2. Partial mediation model (standardized coefficients) standardized regression coefficients are shown next to each path. QoL: quality of life (EORTC QLQ-BR23). 
quality of life as it relates to functioning and symptoms influences depressive symptoms. This observation is consistent with the findings of $\mathrm{Ha}^{8}$ in 2011. In relation to functioning level among the 4 sub-factors of health-related quality of life, depressive symptoms are more serious among 1) patients with poorer body images, 2) patients that hold a more pessimistic view of the future, and 3) patients that show more various and severe breast symptoms and treatment side effects at the symptom level. Breast cancer patients experience a lower quality of life than other cancer patients due to treatment characteristics: that is, the percentage rate for receiving various types of adjuvant therapy including radiation therapy, chemotherapy, and hormone therapy is much higher than for other types of cancers. Moreover, this cancer causes a vast range of symptoms such as deformation at the surgical site, arm swelling, pain, and sensory changes that cause a patient to experience diverse physical side effects. ${ }^{26-28}$ In addition, the cancer gives rise to psychological influences such as fatigue, depression, anxiety, and cognitive impairment. ${ }^{29,30}$ In other words, it is clear that breast cancer patients' health-related quality of life due to these various symptoms and side effects affects depressive symptoms.

Second, findings showed that although self-esteem fully mediated the relationship between quality of life and depressive symptoms, the direct effect of quality life on depressive symptoms was not statistically significant. Hence, for self-esteem, there is only an indirect effect. In other words, breast cancer patients' quality of life does not appear to directly affect depressive symptoms, yet it does have significant influence on depressive symptoms through self-esteem as a mediator. It could be said that low quality of life decreases self-esteem and this low self-esteem intensifies depression, which would be consistent with the results of a study by Tae and Kim. ${ }^{31}$ Their study found that among breast cancer patients' diseaserelated characteristics the physical symptoms of pain and fatigue were associated with self-esteem. It has also been reported that after mastectomy surgery, the sense of loss due to physical changes worsens body image and such damage to body image decreases quality of life. ${ }^{32}$

Tae and $\mathrm{Kim}^{31}$ identified relationships among family support, perceived health state, and self-esteem. The study concluded that the higher breast cancer patients perceived their health state, the higher their self-esteem. Yang and Chung ${ }^{33}$ in their investigation of cancer patients' psychological characteristics and quality of life identified variables that affect quality of life. Cancer patients were found to have high somatization and be prone to depression, anxiety, and hostility. Interestingly, results showed that while depression scores were significantly higher than the normal population control group, self-esteem was significantly lower than the normal popula- tion control group. ${ }^{9,34}$ In a study by Berterö ${ }^{9}$ that investigated the effect of breast cancer patients' self-esteem and quality of life on breast cancer treatment, the effect of self-esteem was found to be an important aspect affecting breast cancer patients' quality of life. Researchers' finding that the effect of breast cancer patients' quality of life on depressive symptoms is fully mediated by self-esteem highlights the role of self-esteem in psychosocial intervention for breast cancer patients. In other words, enhancing self-esteem could lessen stress and prevent progression to more serious depressive symptoms.

Third, optimism was found to partially mediate the relationship between breast cancer patients' quality of life and depression. That is, optimism can be an influencing relationship factor when breast cancer patients' quality of life in respect to functioning and symptoms affects depressive symptoms. It could be concluded that low optimism is one of the major causes of worsening depressive symptoms for patients with low quality of life, which would support the results of a study ${ }^{35}$ on predictors of early breast cancer patients' psychosocial adjustment. In that study, optimism and coping style were major variables improving psychosocial adjustment, and higher optimism scores were found to be associated with higher scores on coping strategies.

Prior research on the degree of depression and anxiety in relation to the body image, self-esteem, and quality of life of breast cancer patients identified factors that predict relationships among the relevant factors of depression and anxiety. The study revealed optimism to be an important factor affecting depressive symptoms; that is to say, previous studies indicate an optimistic view of the future negatively correlates with depression and anxiety. ${ }^{7}$

In another study on predictors of the psychosocial adjustment of women with breast cancer, women with early stage breast cancer who had higher optimism were found to have better psychosocial adjustment. ${ }^{15}$ Also, in Carver et al.s study, ${ }^{36,37}$ the optimism of women with breast cancer was found to significantly correlate with patient coping style, which supports the results of this research. In other words, optimism is concluded to mediate the relationship between quality of life and depressive symptoms as an internal psychological variable, so psychosocial intervention that increases breast cancer patient optimism could prevent depressive symptoms.

Fourth, examination of the relationship between quality of life and depressive symptoms by comparing the two mediating variables found self-esteem to have a full mediating effect whereas optimism had only a partial mediation effect. These results suggest that when quality of life affects depressive symptoms, self-esteem is a more direct causal factor than optimism. Researchers confirmed the relative importance of self- 
esteem and optimism in the process of emerging depressive symptoms by validating their respective effects on mediation.

Providing breast cancer patients with psychological stability, reducing their psychological pain, and enhancing their self-esteem and optimism are meaningful and increase treatment compliance. However, there are limitations to this study's results. First, the age, type of surgery, and disease state of subjects in this study may appear excessively heterogeneous. However, as a multicenter study, recruitment of patients from 3 separate hospitals offered researchers the best opportunity to accumulate a considerable sample of patients. For this reason, research findings are limited to subjects of the study and other variables that might influence a subject's psychiatric condition, this should be investigated in future studies. Second, limitation with this study lies with the generalization of findings because study subjects were breast cancer patients at hospitals from a specific region in South Korea, namely metropolitan Seoul and Gyeonggi Province. Follow-up studies should gather and invite the participation of patients from more varied regions. Third, the mediation model was validated cross-sectionally at a single point in time, making it difficult to state concretely systematic causations in the relationships among quality of life, self-esteem, optimism, and depressive symptoms. However, researchers believe findings add insight and valuable knowledge to the field of breast cancer patient's psychiatric condition. Researchers also suggest that future studies be conducted to validate the mediation effect of self-esteem and optimism systematically, specifically longitudinal studies that consider patients' progress. Lastly, apart from self-esteem and optimism, which were the focus of this research, there are other variables such as hope, resilience, and personal factors that may affect depression and the quality of life. Hence, researchers suggest integrated validation by including several of those various variables in followup studies, which would provide more evidence for psychological intervention.

\section{Acknowledgments}

This work was supported by the 2011 Research Grant of Sookmyung Women's University (1-1103-0397).

\section{REFERENCES}

1. Massie MJ. Prevalence of depression in patients with cancer. J Natl Cancer Inst Monogr 2004;32:57-71.

2. Burgess C, Cornelius V, Love S, Graham J, Richards M, Ramirez A. Depression and anxiety in women with early breast cancer: five year observational cohort study. BMJ 2005;330:702.

3. Somerset W, Stout SC, Miller AH, Musselman D. Breast cancer and depression. Oncology (Williston Park) 2004;18:1021-1034.

4. Nail LM, Winningham ML. Fatigue and weakness in cancer patients: the symptom experience. Semin Oncol Nurs 1995;11:272-278.

5. Mystakidou K, Tsilika E, Parpa E, Katsouda E, Galanos A, Vlahos L. Assessment of anxiety and depression in advanced cancer patients and their relationship with quality of life. Qual Life Res 2005;14:1825-1833.

6. Kim SH, Jun EY, Lee WH. The factors influencing depression in patients with breast cancer. J Korean Oncol Nurs 2006;6:133-142.

7. Choi BJ, Park JH, Choe BM, Han SH, Kim SH. Factors influencing anxiety and depression in breast cancer patients treated with surgery. J Korean Soc Biol Ther Psychiatry 2011;17:87-95.

8. Ha EH. The influence of health related quality of life on depressive symptoms of breast cancer patients. Korean J Woman Psychol 2011;16: 499-515.

9. Berterö CM. Affected self-respect and self-value: The impact of breast cancer treatment on self-esteem and QoL. Psychooncology 2002;11: 356-364.

10. Helms RL, O'Hea EL, Corso M. Body image issues in women with breast cancer. Psychol Health Med 2008;13:313-325.

11. Min SH, Park SY, Kim JI. Effects of cancer-overcome BeHaS exercise program on shoulder joint function, stress, body image and self-esteem in breast cancer patients after surgery. J Korean Acad Fundam Nurs 2011;18:328-336.

12. Scheier MF, Carver CS. Optimism, coping, and health: assessment and implications of generalized outcome expectancies. Health Psychol 1985; 4:219-247.

13. Carver CS, Pozo-Kaderman C, Harris SD, Noriega V, Scheier MF, Robinson DS, et al. Optimism versus pessimism predicts the quality of women's adjustment to early stage breast cancer. Cancer 1994;73:1213-1220.

14. Friedman LC, Kalidas M, Elledge R, Chang J, Romero C, Husain I, et al. Optimism, social support and psychosocial functioning among women with breast cancer. Psychooncology 2006;15:595-603.

15. Westbrook JM. Attachment, optimism, coping, and social support as predictors of psychosocial and psychological adjustment in women with breast cancer women with breast cancer. Santa Barbara: Fielding Graduate University, Unpublished Doctoral dissertation; 2005.

16. Ha EH, Lee SH, Jeong J, Lee HD, Lee JE, Nam SJ, et al. Biopsychosocial predictors of the quality of life in breast cancer patients. J Breast Cancer 2010;13:219-226.

17. Ha EH, Seo JE, Jeong J, Yang JH, Nam SJ, Lee JE, et al. Biopsychosocial predictors of depressive disorder in breast cancer patients. Korean J Clin Psychol 2008;27:961-976.

18. Yun YH, Bae SH, Kang IO, Shin KH, Lee R, Kwon SI, et al. Cross-cultural application of the Korean version of the European Organization for Research and Treatment of Cancer (EORTC) Breast-Cancer-Specific Quality of Life Questionnaire (EORTC QLQ-BR23). Support Care Cancer 2004;12:441-445.

19. Fayers PM, Aaronson NK, Bjordal K, Groenvold M, Curran D, Bottomley A. The EORTC QLQ-C30 Scoring Manual. 3rd Edition. Brussels: EORTC; 2001.

20. Cho MJ, Kim KH. Diagnostic validity of the CES-D (Korean version) in assessment of DSM-III-R major depression. J Korean Neuropsychiatr Assoc 1993;32:381-399.

21. Chon KK, Rhee MK. Preliminary development of Korean version of CES-D. Korean J Clin Psychol 1992;11:65-76.

22. Rosenberg M. Society and the Adolescent Self-Image. Princeton, NJ: Princeton University Press; 1965.

23. Jeon BJ. Self-esteem: a test of its measurability. Yonsei Nonchong 1974; 11:107-130.

24. Park HS, Son JT. A Study on the relationship between optimism and depression in chronic low-back pain patients. J Korean Psychiatr Ment Health Nurs Acad Soc 2001;10:125-136.

25. Curran PJ, West SG, Finch JF. The robustness of test statistics to nonnormality and specification error in confirmatory factor analysis. Psychol Methods 1996;1:16-29.

26. Goss PE, Ingle JN, Martino S, Robert NJ, Muss HB, Piccart MJ, et al. A randomized trial of letrozole in postmenopausal women after five years of tamoxifen therapy for early-stage breast cancer. N Engl J Med 2003; 349:1793-1802.

27. Mock V, Pickett M, Ropka ME, Muscari LE, Stewart KJ, Rhodes VA, et 
al. Fatigue and quality of life outcomes of exercise during cancer treatment. Cancer Pract 2001;9:119-127.

28. Rietman JS, Dijkstra PU, Hoekstra HJ, Eisma WH, Szabo BG, Groothoff JW, et al. Late morbidity after treatment of breast cancer in relation to daily activities and quality of life: a systematic review. Eur J Surg Oncol 2003;29:229-238.

29. Badger TA, Braden CJ, Mishel MH. Depression burden, self-help interventions, and side effect experience in women receiving treatment for breast cancer. Oncol Nurs Forum 2001;28:567-574.

30. Ebright PR, Lyon B. Understanding hope and factors that enhance hope in women with breast cancer. Oncol Nurs Forum 2002;29:561-568.

31. Tae YS, Kim MY. Relationships between family support, perceived health status, and self-esteem in Korean women with breast cancer. Asian Oncol Nurs 2011;11:41-48.

32. Jeong KS, Park GJ. Relationships among body image, self-esteem and health promotion behavior in mastectomy patients. J Korean Oncol
Nurs 2007;7:36-46.

33. Yang JC, Chung WK. Psychology and quality of life in cancer patients on radiation therapy. J Korean Soc Ther Radiol Oncol 2004;22:271-279.

34. Swinney JE. African Americans with cancer: the relationships among self-esteem, locus of control, and health perception. Res Nurs Health 2002;25:371-382.

35. Kim HY, So HS. A structural model for psychosocial adjustment in patients with early breast cancer. J Korean Acad Nurs 2012;42:105-115.

36. Carver CS, Pozo C, Harris SD, Noriega V, Scheier MF, Robinson DS, et al. How coping mediates the effect of optimism on distress: a study of women with early stage breast cancer. J Pers Soc Psychol 1993;65:375390.

37. Carver CS, Smith RG, Antoni MH, Petronis VM, Weiss S, Derhagopian RP. Optimistic personality and psychosocial well-being during treatment predict psychosocial well-being among long-term survivors of breast cancer. Health Psychol 2005;24:508-516. 